Check for updates

Cite this: RSC Adv., 2017, 7, 44254

Received 13th July 2017

Accepted 8th September 2017

DOI: 10.1039/c7ra07714a

rsc.li/rsc-advances

\section{Ultra-high electrical conductivity and superior bendability simultaneously enabled in Ag nanowire based nanocomposites $\uparrow$}

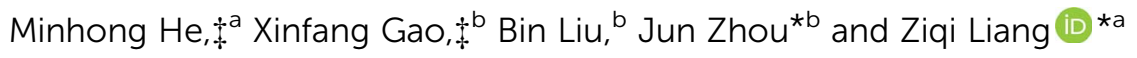 \\ Herein we report the solution fabrication of highly conductive and bendable conductors based on $\mathrm{Ag}$ \\ nanowires (NWs) embedded within a polyvinylidene fluoride matrix. A typical percolation behavior of \\ electrical conductivity is observed due to the formation of a conductive network. The highest \\ conductivity, $1.77 \times 10^{4} \mathrm{~S} \mathrm{~cm}^{-1}$, is achieved at $80 \mathrm{wt} \% \mathrm{Ag} \mathrm{NWs}$ and it remains nearly unchanged after \\ 1000 bending events
}

With rapid development of bendable and wearable electronics and sensors, there is a growing demand for flexible conductors, ${ }^{1,2}$ which play a significant role as interconnects or electrodes in a wide range of revolutionary functional devices ranging from roll-up displays, ${ }^{3,4}$ and wearable electronic equipment, ${ }^{5,6}$ to artificial and smart skin. ${ }^{7-11}$ Indeed, high mechanical strength and large electrical conductivity of conductors are required in these numerous applications. ${ }^{12-18}$ Promising candidates that meet the above requirements are nanocomposites with inorganic conductive fillers embedded in an organic matrix. In this regard, an effective electron pathway can be established by forming the network of fillers, while the matrix provides good mechanical properties. However, most of such nanocomposites suffer from poor electrical conductivity because of the limited filling fraction. A large fraction of conductive fillers would otherwise inevitably undermine the elasticity, deformability and processability of the resulting nanocomposites. For example, $\mathrm{Xu}$ and $\mathrm{Zhu}$ reported that the highest filling fraction of metal nanowires (NWs) were estimated to be less than $\sim 70 \mathrm{wt} \%$ in the nanocomposites based on polydimethylsiloxane (PDMS) matrix, showing the best conductivity of $8130 \mathrm{~S} \mathrm{~cm}^{-1} \cdot{ }^{19}$ Above the upper limit fraction, the samples would be easily cracked when peeled off from the substrate and prone to wear and tear during practical operation. It is thus highly desirable to develop such nanocomposites that

\footnotetext{
${ }^{a}$ Department of Materials Science, Fudan University, Shanghai 200433, China. E-mail: zqliang@fudan.edu.cn

${ }^{b}$ Center for Phononics and Thermal Energy Science, China-EU Joint Center for Nanophononics, Shanghai Key Laboratory of Special Artificial Microstructure Materials and Technology, School of Physics Science and Engineering, Tongji University, Shanghai 200092, China. E-mail: zhoujunzhou@tongji.edu.cn

$\dagger$ Electronic supplementary information (ESI) available. See DOI: $10.1039 / \mathrm{c} 7 \mathrm{ra} 07714 \mathrm{a}$

$\$$ Authors contributed equally to this work.
}

possess good conductivity and mechanical flexibility simultaneously.

It is straightforward to choose silver (Ag) as the conductive filler due to its excellent electrical conductivity and malleability. ${ }^{20,21}$ Several research groups have made the nanocomposites with Ag NWs dispersed in PDMS matrix. For instance, Huang et al. showed that Ag NWs were embedded into the PDMS interlayer and formed a sandwich structure, yielding an electrical conductivity as high as $1.52 \times 10^{4} \mathrm{~S} \mathrm{~cm}^{-1}$. $^{22}$ Park et al. prepared a conductive nanocomposite mat by coating $\mathrm{Ag}$ nanoparticles onto rubber fibres and acquired the highest conductivity of $\sim 5400 \mathrm{~S} \mathrm{~cm}^{-1}$ in the presence of large stretching deformation. ${ }^{23}$ These recent advances point to growing opportunities for highly conductive and flexible conductors based on Ag NWs with multi-layer structures in which the binding force between the layers is however poor, resulting in potential risk of damage after repeated bending.

In this work, we solution fabricated such nanocomposites that exhibited both superior electrical conductivity and excellent mechanical bending properties by dispersing Ag NWs uniformly into the polymeric matrix. Polyvinylidene fluoride (PVDF) was selected as the matrix material owing to its high temperature tolerance along with good thermal stability, plasticity, film-forming, mechanical strength and chemical resistance, ${ }^{24}$ which can be potentially applied to wearable electronic equipment. ${ }^{25}$ We made a range of nanocomposites with different filling fractions of $\mathrm{Ag}$ NWs and characterized their electrical conductivities and bendabilities, accordingly. At the content of $80 \mathrm{wt} \% \mathrm{Ag}$ NWs in nanocomposites, the obtained conductivities are as high as $1.77 \times 10^{4} \mathrm{~S} \mathrm{~cm}^{-1}$ which is among the best reported values and more importantly preserved with repeated bending cycles.

The Ag NWs/PVDF nanocomposites of several micrometres thick were attained by mixing Ag NWs and PVDF powders in dimethylformamide (DMF) solution and subsequently drying in 
aluminium molds within a $\mathrm{N}_{2}$-filled glovebox. The experimental details are described in ESI. $\dagger$ Their phase structures were inspected by X-ray diffraction (XRD) method. As shown in Fig. 1, the nanocomposites show strong diffraction peaks at 38.1, 44.3, 64.6 and 77.4 degrees, which correspond to the reflections from (111), (200), (220), and (311) planes of Ag crystal structure, ${ }^{26}$ respectively. These peaks are progressively enhanced in intensity when the mass fraction of Ag NWs increases from $5 \mathrm{wt} \%$ to $80 \mathrm{wt} \%$. On the other hand, the absence of peaks at 32.8, 38.0, 54.8 , and 65.4 degrees which are related to $\mathrm{Ag}_{2} \mathrm{O}$ suggest that the oxidization of $\mathrm{Ag}$ is prevented during the solution mixing process.

Typical photographs of as-fabricated Ag NWs/PVDF nanocomposite film are displayed in Fig. 2a, showing that the film is free-standing and has a smooth surface with an area of $4.1 \mathrm{~cm}^{2}$. Moreover, the film is easily bent without breaking. To quantify its superior bendability, the insets show photographs of a film inside a tube with a radius size of $4.7 \mathrm{~mm}$, thus demonstrating its desired relevance for flexible and wearable modules. Fieldemission scanning electron microscopy (FE-SEM) was further conducted to examine the morphology and nanostructures of the nanocomposites, and their typical top-view images are presented in Fig. 2b-f. It is clearly seen that Ag NWs are randomly distributed over the entire region of PVDF, in which the existence of high aspect ratio Ag NWs is evident and typical three-dimensional percolation networks are available dependent upon the filling fractions of Ag NWs.

At a low filling fraction of $5 \mathrm{wt} \%$, the majority of $\mathrm{Ag}$ NWs are isolated in nanocomposites as shown in Fig. 2b. As the fraction is increased to $15 \mathrm{wt} \%$, a dense distribution of nanowire conjunctions is found while a connecting network is formed (Fig. 2c). This network is usually named as infinite cluster in percolation theory. Furthermore, such a network of Ag NWs in nanocomposites becomes more visible when the filling fraction continues to increase and the resulting electrical pathway is favourable for electron transport (Fig. 2d-f). It can be briefly concluded that with increasing filling fraction, more transport pathways are generated due to the presence of more conductive filler, which is advantageous for obtaining higher conductivity.

The filling fraction, distribution, aspect ratio and alignment of $\mathrm{Ag}$ NWs in nanocomposites would greatly affect electrical

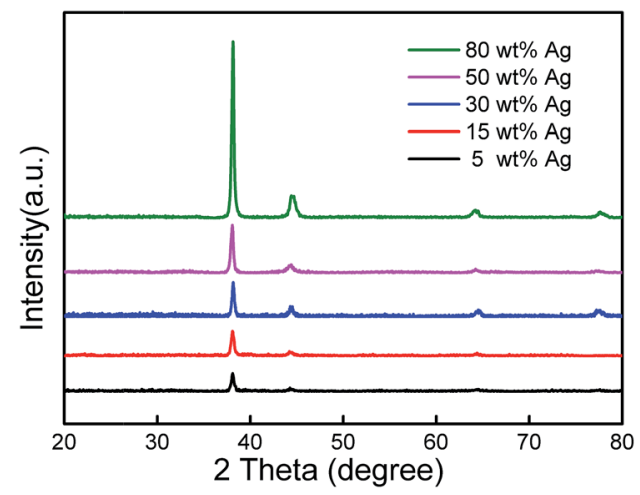

Fig. 1 XRD patterns of Ag NWs/PVDF nanocomposites with different filling fractions.

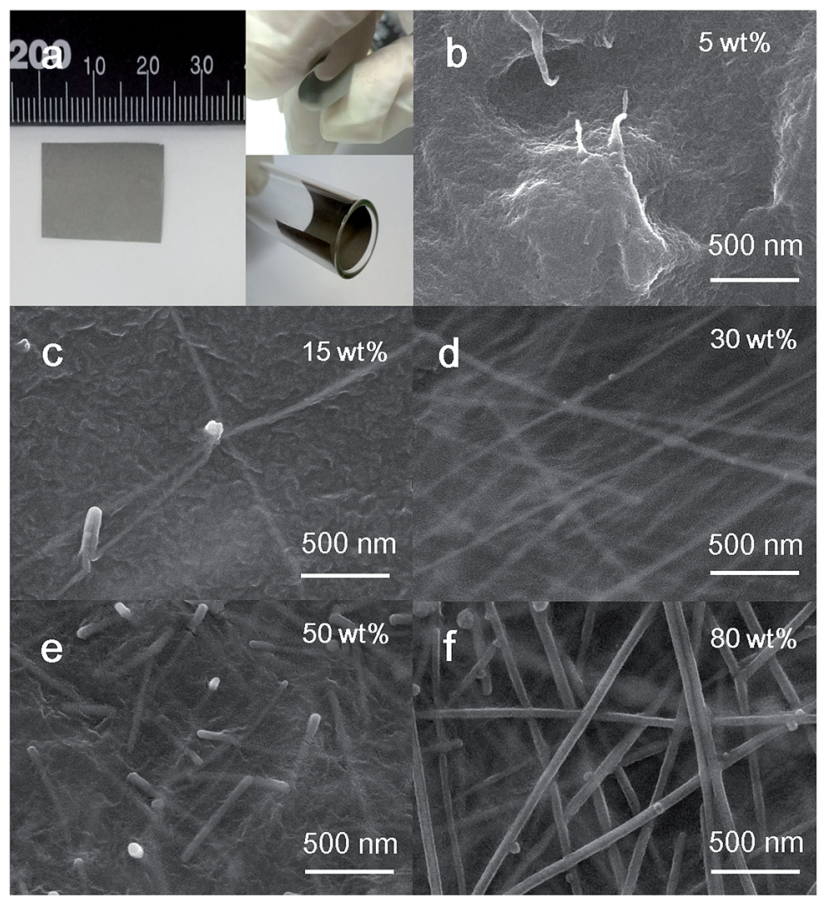

Fig. 2 (a) Typical photographs of highly bendable Ag NWs/PVDF film. $(b-f)$ Top-view SEM images of the nanocomposites with different filling fractions of Ag NWs.

conductivity $(\sigma)$. Fig. 3a shows the room-temperature $\sigma$ dependences of Ag NWs/PVDF nanocomposite films on the filling fraction of Ag NWs. As shown in Fig. 3a and Table S1, $\uparrow \sigma$ increases slowly from $3.68 \times 10^{-6} \mathrm{~S} \mathrm{~cm}^{-1}$ at $5 \mathrm{wt} \% \mathrm{Ag}$ NWs to $5.00 \times 10^{2} \mathrm{~S} \mathrm{~cm}^{-1}$ at $40 \mathrm{wt} \%$, then significantly from $1.55 \times$ $10^{3} \mathrm{~S} \mathrm{~cm}^{-1}$ at $50 \mathrm{wt} \%$ up to $1.77 \times 10^{4} \mathrm{~S} \mathrm{~cm}^{-1}$ at $80 \mathrm{wt} \%$. This phenomenon can be rationalized as the formation and expansion of infinite cluster of Ag NWs. Fig. 3c and d illustrate the electrical conduction mechanism within nanocomposite films with low and high filling fractions of $\mathrm{Ag}$ NWs, respectively. According to the percolation theory, when the volume fraction $(\phi)$ of $\mathrm{Ag}$ NWs is smaller than a critical value which is known as the critical volume fraction $\left(\phi_{\mathrm{c}}\right)$, only isolated and finite clusters of $\mathrm{Ag}$ NWs exist in nanocomposites. Thus, electrons cannot transport from left to right side as shown in Fig. 3c. When $\phi$ is larger than $\phi_{\mathrm{c}}$, isolated clusters merged into a larger cluster, which is termed as infinite cluster in percolation theory. Therefore, electrons are able to transport from left to right side along a connected pathway as shown in Fig. 3d. Further increasing of $\phi$ raises the number of connected pathways which results in an increase of electrical conductivity.

The $\sigma$ values of nanocomposites can be fitted by using a phenomenological relation as shown in eqn (1) based on power law dependence where $t$ is the exponent factor. The fitting shows $\phi_{\mathrm{c}}=0.28 \mathrm{vol} \%$ which corresponds to a filling fraction $1.63 \mathrm{wt} \%$, and $t=2$ in three-dimensional (3D) system. ${ }^{27}$ The fitted curves are in good agreement with the experimental results (as shown in Fig. 3a), thus providing strong evidence for the formation of a 3D percolation network, which favors electron transport. The aspect ratio of the $\mathrm{Ag}$ NWs is greater than 


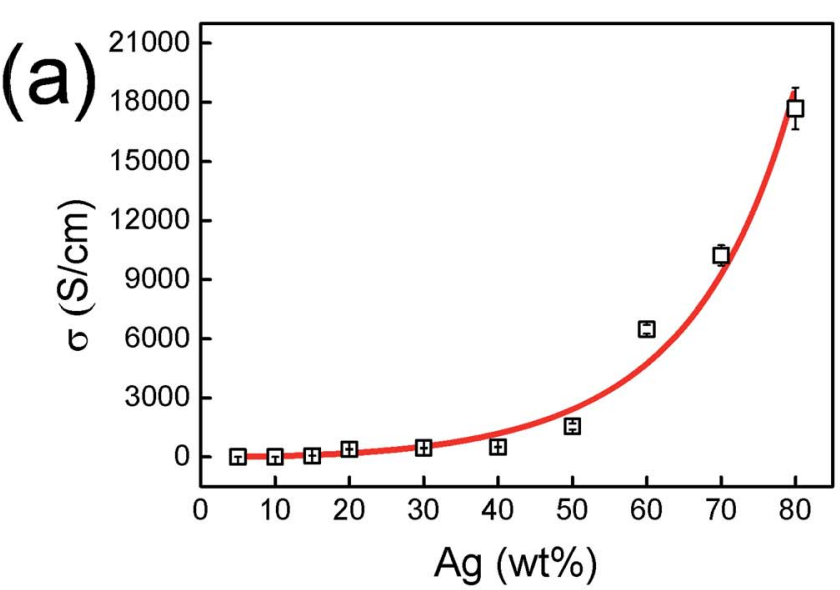

(b)
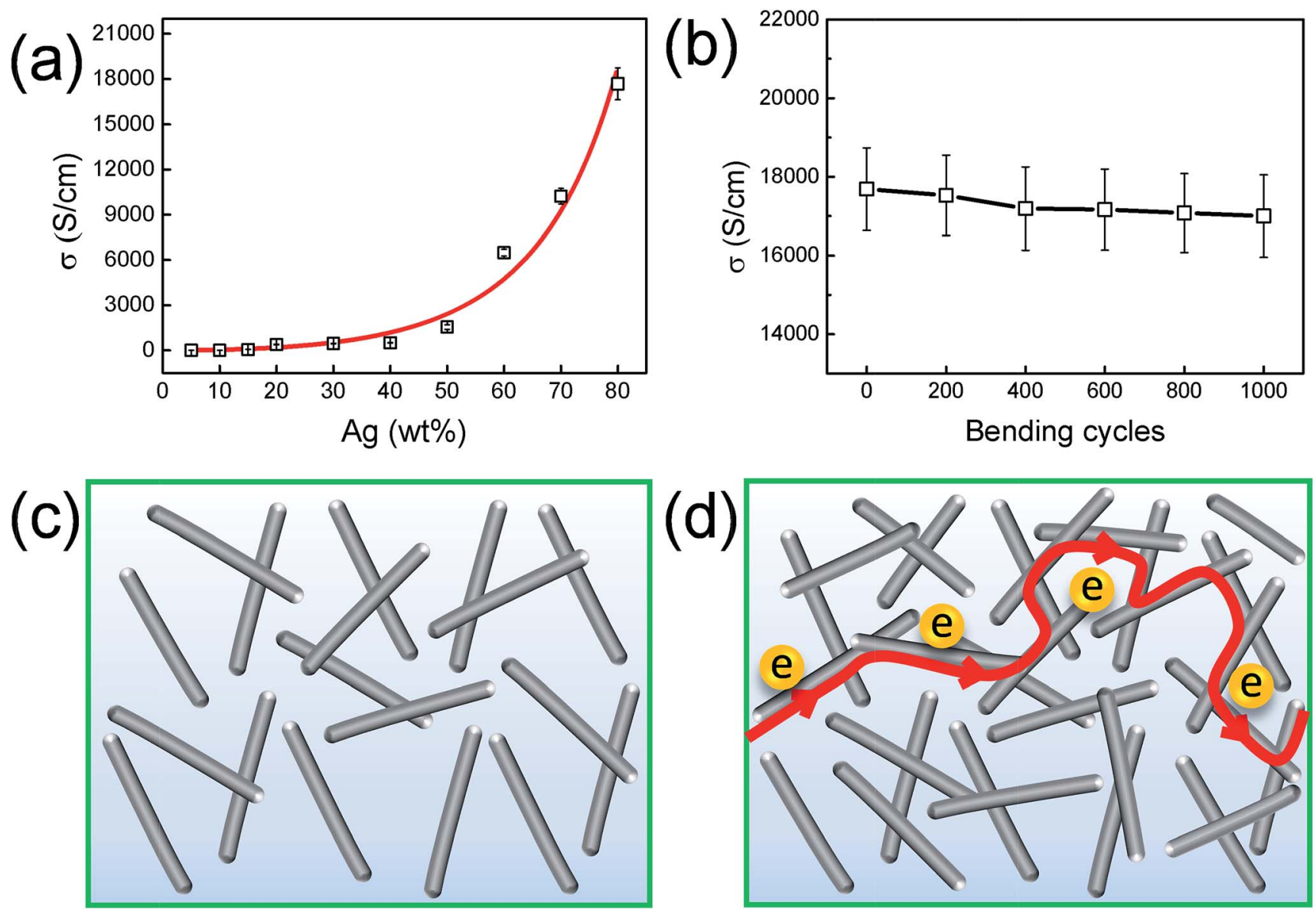

Fig. 3 (a) Filling fraction dependences of electrical conductivity $(\sigma)$ of the Ag NWs/PVDF nanocomposites. (b) Conductivity of the Ag NWs (80 wt\%)/PVDF film versus the bending cycles from flat to a bending radius of $2.5 \mathrm{~mm}$ and back to flat. The points are experimental data and the fitted line in (a) is the corresponding numerical simulated results while the line in (b) is guideline. (c, d) Schematic illustrating the formation of conductive Ag NWs channel, which is dependent on the volume fraction of Ag NWs in nanocomposite films. A conductive pathway of Ag NWs is marked in red in (d).

300 because the averaged diameter of NWs is $60 \mathrm{~nm}$ and the lengths of NWs are in the range of $20-80 \mu \mathrm{m}$. Based on these parameters, the numerical simulated $\phi_{\mathrm{c}}$ is around $0.2 \mathrm{vol} \%$ by using the Monte-Carlo method, ${ }^{28}$ which is very close to the fitted value in eqn (1).

$$
\sigma \propto\left(\phi-\phi_{\mathrm{c}}\right)^{t}
$$

In addition, we investigated the influence of mechanical bending on electrical conductivity of Ag NWs $(80 \mathrm{wt} \%) / \mathrm{PVDF}$ nanocomposite film. It is clearly seen from Fig. $3 \mathrm{~b}$ that the conductivity of $\mathrm{Ag}$ NWs $(80 \mathrm{wt} \%) / \mathrm{PVDF}$ film remains little changed when bending to a radius of $2.5 \mathrm{~mm}$ and then releasing back to flat. With the increase of the number of bending events, conductivity decreased slightly, even after 1000 bending events, the ultra-high electrical conductivity of Ag NWs ( $80 \mathrm{wt} \%$ )/PVDF film is maintained constant with only $\sim 4 \%$ loss, indicating that the nanocomposite film possesses outstanding electrical conductivity and flexibility simultaneously.

Lastly, we demonstrated the application of such nanocomposite films for flexible conductors. We constructed a prototypic electrical circuit experiment by cutting as- fabricated Ag NWs (5-80 wt\%)/PVDF films into a shape that is connected to the circuit, as shown in Fig. 4a-f. The samples with different filling fractions were connected while observing the changes of the diode luminance. It is found that when the filling fraction is $5 \mathrm{wt} \%$ (Fig. 4a), the diode is off, indicating that nanocomposites is almost insulator, consistent with the very low conductivity of $3.7 \times 10^{-6} \mathrm{~S} \mathrm{~cm}^{-1}$ as shown in Fig. 3a. When the filling fraction continues to increase, the diode remains lit. It is explicitly seen from Fig. $4 \mathrm{~b}-\mathrm{f}$ that the luminance increases with the increasing filling fraction. This phenomenon confirms that as the filling fraction increases, a better conductive grid is formed and hence the conductivity is increased. At the highest fraction of $80 \mathrm{wt} \% \mathrm{Ag}$ NWs, the composite film displays the brightest diode luminesce at the bending state, indicating its best conductivity (Fig. 4g).

Also, we bended the film to control the diode by disconnecting and reconnecting the circuit. Fig. $4 \mathrm{~h}$ and $\mathrm{i}$ and Movie S1 in the ESI $\uparrow$ illustrate such processes of the Ag NWs (80 wt\%)/PVDF film. Remarkably, the composite film presents excellent bendability and deformability while preserving good conductivity. 


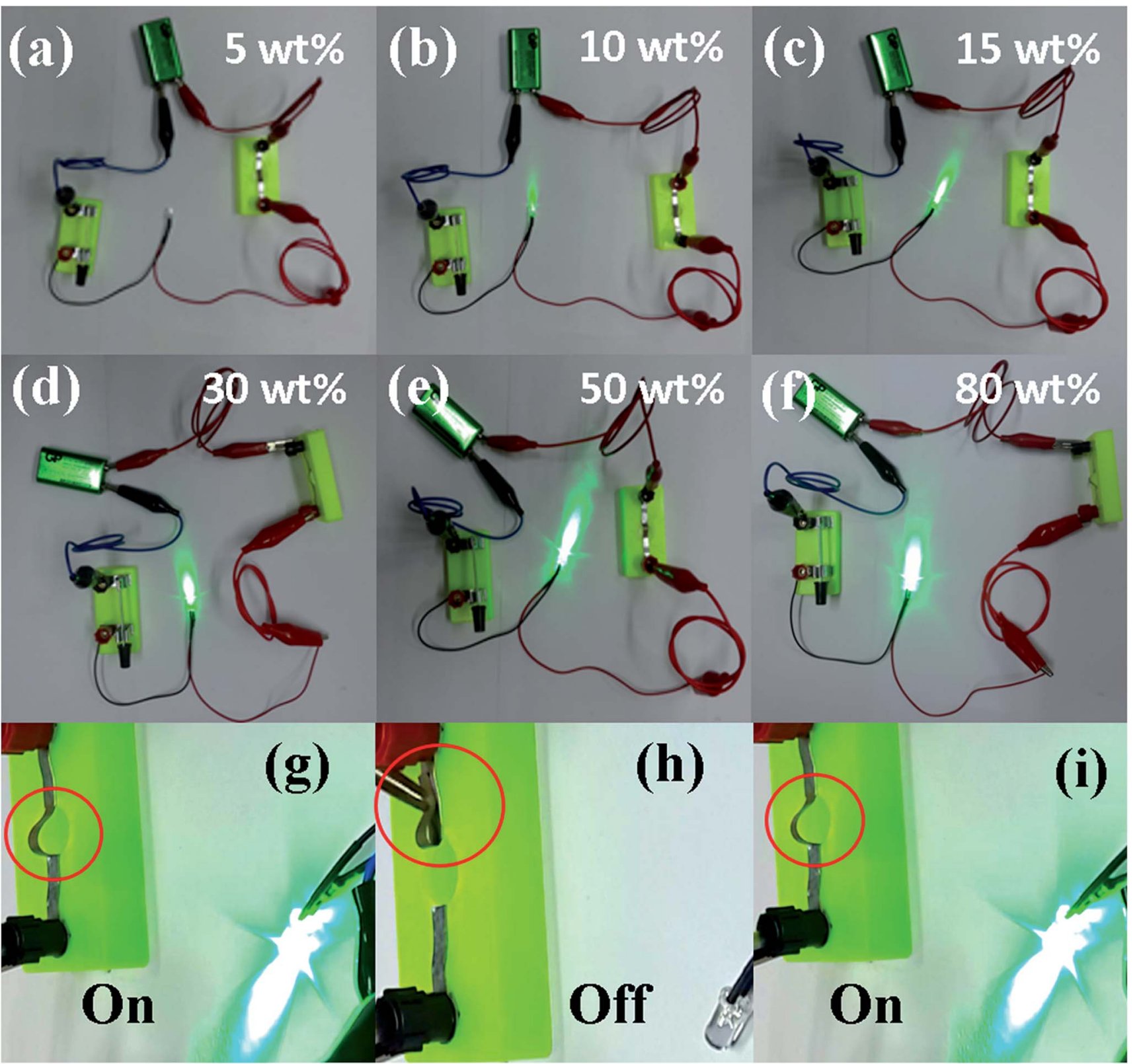

Fig. 4 Demonstration experiments of the Ag NWs/PVDF nanocomposites. (a-f) With increasing filling fraction, the luminance of the diode is notably enhanced. ( $g-i)$ The maintained high conductivity, when bending, of the optimal Ag NWs ( 80 wt\%)/PVDF film as indicated by red cycle.

In conclusion, we have successfully fabricated ultra-highly conductive and flexible nanocomposites by homogeneously embedding Ag NWs network within PVDF matrix. A typical percolation behavior of electrical conductivity is observed due to the formation of conductive network. The critical volume fraction of percolation is found to be as low as $0.28 \mathrm{vol} \%$ which is in excellent agreement with the numerical simulation results. We also find that a quadratic dependence of electrical conductivity on $\phi-\phi_{c}$, which is consistent with the electrical percolation theory. When the filling fraction of $\mathrm{Ag}$ NWs is $80 \mathrm{wt} \%$, an ultra-high electrical conductivity of $1.77 \times$ $10^{4} \mathrm{~S} \mathrm{~cm}^{-1}$ is obtained, which is among the highest value of silver based composites. ${ }^{19,22,23}$ The samples show superior stability because the high electrical conductivity is almost unchanged after 1000 bending events. Therefore, our study demonstrates a promising fabrication method of highly conductive and flexible conductors for the potential applications of wearable electronic devices.

\section{Conflicts of interest}

There are no conflicts to declare.

\section{Acknowledgements}

This work is sponsored by the National Natural Science Foundation of China (NSFC) under grant No. 51673044 (Z. L.). J. Z. acknowledges the support from the program for Professor of 
Special Appointment (Eastern Scholar) at Shanghai Institutions of Higher Learning (Grant No. TP2014012).

\section{References}

1 M. Melzer, J. I. Mönch, D. Makarov, Y. Zabila, G. S. C. Bermúdez, D. Karnaushenko, S. Baunack, F. Bahr, C. Yan, M. Kaltenbrunner and O. G. Schmidt, Adv. Mater., 2015, 27, 1274-1280.

2 W. Lin, S. Liu, T. Gong, Q. Zhao and W. Huang, Adv. Mater., 2014, 26, 570-606.

3 L. Zhou, A. Wanga, S. Wu, J. Sun, S. Park and T. N. Jackson, Appl. Phys. Lett., 2006, 88, 083502.

4 S. Kim, H.-J. Kwon, S. Lee, H. Shim, Y. Chun, W. Choi, J. Kwack, D. Han, M.-S. Song, S. Kim, S. Mohannadi, I.-S. Kee and S. Y. Lee, Adv. Mater., 2011, 23, 3511-3516.

5 S. Chen, Z. Lou, D. Chen, K. Jiang and G. Shen, Adv. Mater. Technol., 2016, 1, 1600136.

6 Q. Wu and J. Hu, Smart Mater. Struct., 2017, 26, 045037045044.

7 J. Ge, L. Sun, F. R. Zhang, Y. Zhang, L. A. Shi, H. Y. Zhao, H. W. Zhu, H. L. Jiang and S. H. Yu, Adv. Mater., 2016, 28, 722-728.

8 I. You, B. Kim, J. Park, K. Koh, S. Shin, S. Jung and U. Jeong, Adv. Mater., 2016, 28, 6359-6364.

9 M. Kaltenbrunner, T. Sekitani, J. Reeder, T. Yokota, K. Kuribara, T. Tokuhara, M. Drack, R. Schwodiauer, I. Graz, S. Bauer-Gogonea, S. Bauer and T. Someya, Nature, 2013, 499, 458-463.

10 J. Y. Sun, C. Keplinger, G. M. Whitesides and Z. Suo, Adv. Mater., 2014, 26, 7608-7614.

11 S. Park, H. Kim, M. Vosgueritchian, S. Cheon, H. Kim, J. H. Koo, T. R. Kim, S. Lee, G. Schwartz, H. Chang and Z. Bao, Adv. Mater., 2014, 26, 7324-7332.

12 Y. Hu, T. Zhao, P. Zhu, Y. Zhu, X. Shuai, X. Liang, R. Sun, D. D. Lu and C.-P. Wong, J. Mater. Chem. C, 2016, 4, 58395848.
13 T. Cheng, Y. Zhang, W.-Y. Lai and W. Huang, Adv. Mater., 2015, 27, 3349-3376.

14 T. Q. Trung and N.-E. Lee, J. Mater. Chem. C, 2017, 5, 22022222.

15 J. Liang, L. Li, K. Tong, Z. Ren, W. Hu, X. Niu, Y. Chen and Q. Pei, ACS Nano, 2014, 8, 1590-1600.

16 H. Wu, Y. A. Huang, F. Xu, Y. Duan and Z. Yin, Adv. Mater., 2016, 28, 9881-9919.

17 H. Lee, I. Kim, M. Kim and H. Lee, Nanoscale, 2016, 8, 17891822.

18 Z. Yang, J. Deng, X. Sun, H. Li and H. Peng, Adv. Mater., 2014, 26, 2643-2647.

19 F. Xu and Y. Zhu, Adv. Mater., 2012, 24, 5117-5122.

20 M. Bharti, A. Singh, S. Samanta, A. K. Debnath, D. K. Aswal, K. P. Muthe and S. C. Gadkari, Energy Convers. Manage., 2017, 144, 143-152.

21 L. Hu, H. S. Kim, J.-Y. Lee, P. Peumans and Y. Cui, ACS Nano, 2010, 4, 2955-2963.

22 G.-W. Huang, H.-M. Xiao and S.-Y. Fu, Sci. Rep., 2015, 5, 13971.

23 M. Park, J. Im, M. Shin, Y. Min, J. Park, H. Cho, S. Park, M.-B. Shim, S. Jeon, D.-Y. Chung, J. Bae, J. Park, U. Jeong and K. Kim, Nat. Nanotechnol., 2012, 7, 803-809.

24 C. Silvestre, D. Duraccio and S. Cimmino, J. Mater. Chem. A, 2017, 5, 10470-10479.

25 P. Martins, A. C. Lopes and S. Lanceros-Mendez, Prog. Polym. Sci., 2014, 39, 683-706.

26 J. Ma, K. Wang and M. Zhan, ACS Appl. Mater. Interfaces, 2015, 7, 16027-16039.

27 N. Shenogina, S. Shenogin, L. Xue and P. Keblinski, Appl. Phys. Lett., 2005, 87, 133106.

28 M. Foygel, R. D. Morris, D. Anez, S. French and V. L. Sobolev, Phys. Rev. B: Condens. Matter Mater. Phys., 2005, 71, 104201. 